\title{
Comparison of Ability of Platelet-rich Fibrin vs CollaPlug in maintaining the Buccal Bone Height of Sockets following Extractions in 20 Patients
}

\author{
${ }^{1} \mathrm{G}$ Madhan, ${ }^{2}$ Madhumati Singh
}

\begin{abstract}
Introduction: The preservation of bone volume immediately after tooth removal is necessary to optimize the success of implant placement in terms of esthetics and function. The objectives of this study were to compare the ability of Choukroun's platelet-rich fibrin (PRF) versus CollaPlug (Zimmer) in maintaining the buccal bone height of sockets following extractions in patients.
\end{abstract}

Materials and methods: Twenty patients who required tooth extraction and implant placement were enrolled in this study. The patients were randomly divided into two groups. They are group I PRF group and group II CollaPlug group. The vertical buccal crestal bone heights were measured immediately after extraction and 4 months postextraction and implants were placed.

Results: The buccal crestal bone level in the CollaPlug group had a baseline mean of $4.67 \pm 0.54$ and a postmean of $6.98 \pm$ 0.60 , whereas in the PRF group baseline mean was $5.43 \pm$ 0.47 and postmean was $6.93 \pm 0.55$. The bone resorption was found in both the groups $(2.31 \mathrm{~mm}$ for CollaPlug and $1.5 \mathrm{~mm}$ for PRF), in agreement with previous studies. However, there was increased bone loss in CollaPlug group compared with PRF group, which was found statistically significant

Conclusion: In conclusion, within the limits of the present study, the two tested socket preservation materials seem to be effective in the treatment of extraction sockets, even though the design of the study did not allow us to evaluate to what extent the clinical improvement could be attributed to the PRF per se, since a negative control was not included in this investigation. However, preparation of PRF is not very cumbersome and inexpensive, which makes it a better socket preservation material than CollaPlug.

Keywords: Choukroun's platelet-rich fibrin, CollaPlug (Zimmer), Vertical buccal crestal bone heights.

How to cite this article: Madhan G, Singh M. Comparison of Ability of Platelet-rich Fibrin vs CollaPlug in maintaining the Buccal Bone Height of Sockets following Extractions in 20 Patients. J Health Sci Res 2017;8(1):1-6.

Source of support: Nil

Conflict of interest: None

\footnotetext{
${ }^{1}$ Reader, ${ }^{2}$ Professor and Head

1,2Department of Oral and Maxillofacial Surgery, RajaRajeswari Dental College \& Hospital, Bengaluru, Karnataka, India

Corresponding Author: G Madhan, Reader, Department of Oral and Maxillofacial Surgery, RajaRajeswari Dental College and Hospital, Bengaluru, Karnataka, India, Phone: +91-8025420818 e-mail: drmadhang@gmail.com
}

\section{INTRODUCTION}

Tooth replacement with dental implant-supported restoration is currently a well-accepted treatment alternative to traditional fixed and removable dental prostheses. Short- and long-term clinical studies have reported similar if not higher prognosis for these restorations. The challenge is that even the current dental implant design does not mimic natural root form, particularly in an immediate implant placement situation. The incongruity between the shape of the implant body and that of the socket wall may lead to gaps between the bone and the implant. Thus, following tooth extraction, there would not be sufficient bone-implant surface contact for more predictable osseointegration, and a poorer peri-implant soft and hard tissue contours following healing. ${ }^{2,3,5-7}$ Secondly, studies ${ }^{1,5,8,10}$ have shown immediate implant placement has also not prevented alveolar bone loss. Hence, there is a significant interest in developing new surgical techniques and biomaterials to prevent alveolar bone loss following tooth extraction. ${ }^{1}$

The healing of extraction wound has been studied extensively both histologically and radiographically in humans. It is currently understood that during the first 8 weeks following extraction there is a marked osteoclastic bone resorption that takes place on the surface of the residual ridge particularly the coronal part of the buccal bone wall and there is reduction of buccal crest in both the vertical and horizontal planes, with the degree of horizontal bone loss typically exceeding the degree of vertical bone loss. ${ }^{2-7}$

Alveolar ridge preservation (ARP) is any procedure undertaken at the time of or following an extraction that is designed to minimize external resorption of the ridge and to maximize bone formation within the socket. Current methods to prevent ridge resorption include the use of various particulate grafts and membranes manufactured from various materials. Most of these materials have been shown to be osteoconductive, providing a scaffold for the osteoblasts to migrate and form bone. ${ }^{8-10}$

Insertion of a filler material (best being autologous bone) into the socket is considered important to preserve as much bone as possible. Serino et al used 
a commercially available bioabsorbable sponge of polylactide-polyglycolide, which served as a support to prevent the collapse of the surrounding soft tissue into the socket during the healing process. A similar product is available commercially as collagen plug (CollaPlug, Zimmer Dental). This was placed into the socket without raising a flap. ${ }^{11,12}$

The importance of growth factor in enhancing wound healing has become the focus of research in the present day, as they hasten the healing process. They are osteoinductive unlike the other bone grafts which are osteoconductive and remain unresorbed in the grafted site. ${ }^{13,14}$

\section{Platelet-rich Fibrin}

Platelet-rich fibrin (PRF) represents a new step in the platelet gel therapeutic concept with simplified processing minus artificial biochemical modification. Unlike other platelet concentrates, this technique requires neither anticoagulants nor bovine thrombin (nor any other gelifying agent). It is just centrifuged natural blood without additives. ${ }^{15}$

Developed in France by Choukroun et al in 2001, the PRF production protocol attempts to accumulate platelets and released cytokines in a fibrin clot. Though platelets and leukocyte cytokines play an important part in the biology of this biomaterial, the fibrin matrix supporting them certainly constitutes the determining element responsible for the real therapeutic potential of PRF. ${ }^{15-18}$

Clinical and histological effectiveness of treatment of an extraction socket with Choukroun's PRF versus CollaPlug (Zimmer) as a socket preservation material was done in the present study.

\section{OBJECTIVE OF THE STUDY}

The objective of the study was to evaluate and compare the bone dimensional changes clinically in extraction sockets with growth factor-enhanced fibrin matrix (Choukroun's PRF) against bioabsorbable collagen matrix (CollaPlug) and to evaluate bone regeneration capacity of PRF.

\section{MATERIALS AND METHODS}

\section{Patient Selection}

All patients above 18 years of either sex reporting to the Department of Oral and Maxillofacial Surgery, RajaRajeswari Dental College and Hospital, Bengaluru, requiring tooth extraction followed by implant placement were selected for this study. Written informed consent was obtained from those who agreed for participation. Ethical clearance was taken for the study. Our inclusion criteria were systemically healthy subjects, sockets with intact four-wall configuration evaluated clinically by probing, healthy periodontium, and presence of at least one tooth adjacent to socket for the preparation and adaptation of acrylic stent for measurements. Subjects with any major systemic illness, acutely infected tooth at the time of extraction, and a history of head and neck radiation therapy and cigarette smokers were not included. Twenty patients fulfilling the abovementioned criteria were selected and randomly assigned to one of the two groups.

According to the protocol for presurgical preparation, upper and lower impressions were taken to obtain a working model. Acrylic stents were made on the working model including at least one tooth adjacent to the tooth to be extracted to serve as a fixed reference guide for the vertical measurements done using a standardized periodontal probe; the measurements were done with base of stent as reference. Measurements were done before extraction, immediately after extraction, and at 4 th month during reentry.

Group I-Choukroun's PRF: To produce PRF, $10 \mathrm{~mL}$ of blood was drawn from each patient of the group by venipuncture, collected into a sterile test tube without any anticoagulant, and centrifuged in a centrifuge machine (REMI Laboratories, Mumbai) for 9 minutes at 2,700 rpm. This resulted in separation of three basic fractions: Red blood cells (RBCs), PRF, and platelet-poor plasma (PPP). Because of differential densities, the RBC layer forms the lowest layer, the PRP layer is the middle one, and the PPP layer is at the top. Platelet-rich fibrin layer was taken out using a sterile tweezer at the time of surgery and was placed into the socket.

Group II—CollaPlug: In group II patients CollaPlug (Zimmer) was placed into the socket.

Atraumatic extraction was done using luxators. Immediate postextraction measurements of vertical distance of crestal bone buccally from the apical end of the stent were done using periodontal probe as a reference. Wound closure was done after placing either PRF or CollaPlug.

Follow-up was done after 4 months for a reentry procedure and implant placement. For the measurements, previously used acrylic stents were placed and all the measurements were repeated.

\section{RESULTS}

The present clinical study was carried out to compare the clinical effectiveness of Choukroun's PRF and CollaPlug (Zimmer) in preserving the dimensions of extraction socket during the healing period of 4 months. 
Comparison of Ability of Platelet-rich Fibrin vs CollaPlug in maintaining the Buccal Bone Height of Sockets

Table 1: Descriptive statistics of buccal crestal bone level among the two groups in baseline and post-ARP tests

\begin{tabular}{|c|c|c|c|c|c|c|c|c|c|c|}
\hline \multirow[b]{2}{*}{ Group } & \multicolumn{2}{|c|}{ Min } & \multicolumn{2}{|c|}{ Max } & \multicolumn{2}{|c|}{ Mean } & \multicolumn{2}{|c|}{ Std. Dev } & \multicolumn{2}{|c|}{ Std. Error (mean } \\
\hline & Baseline & Post & Baseline & Post & Baseline & Post & Baseline & Post & Baseline & Post \\
\hline CollaPlug & 3.33 & 5.00 & 8.33 & 10.33 & 4.67 & 6.98 & 1.71 & 1.91 & 0.54 & 0.60 \\
\hline PRF & 3.33 & 5.33 & 7.33 & 10.33 & 5.43 & 6.93 & 1.48 & 1.72 & 0.47 & 0.55 \\
\hline
\end{tabular}

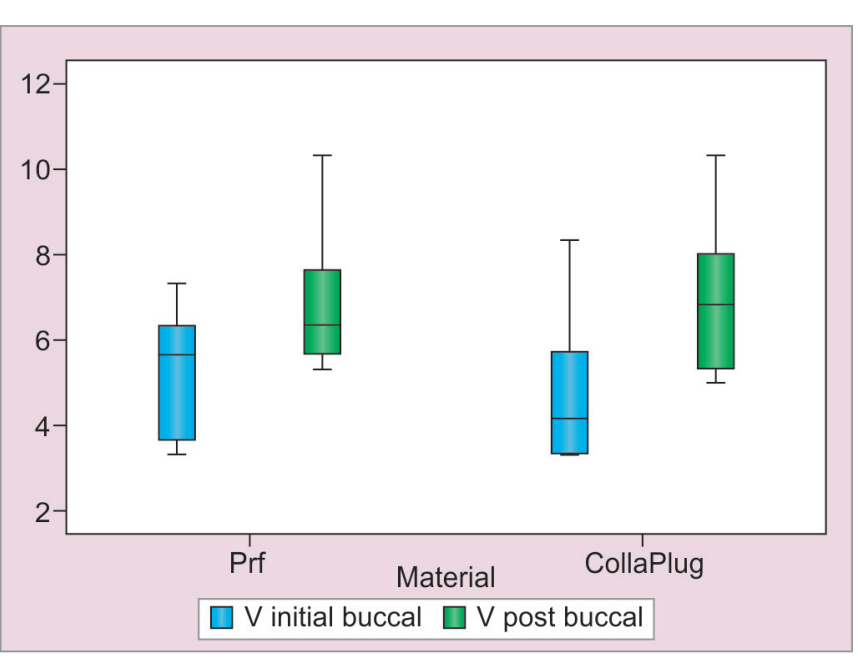

Graph 1: Boxplot of buccal crestal bone level baseline and post-ARP in the two groups

A total of 20 patients (mean age $24.7 \pm 1.3$ years) satisfying the selection criteria were included in the study and randomly allocated into PRF group and CollaPlug group (10 patients in each group). The gender distribution was similar between the two groups. The study involved only patients requiring maxillary anterior teeth extractions and replacement with an implant-supported prosthesis. At the extraction site, the buccal crestal bone level from the base of stent was recorded at baseline (at the time of extraction) and post-ARP, at 4 months.

The parameters recorded were subjected to the following statistical analysis:

- Descriptive statistics like minimum (min), maximum (max), mean, standard error (std. error) of mean, and standard deviation (std. dev) are given to make some general observations about the data of clinical parameters among the two groups in baseline test and post-ARP.

- Analysis of covariance (ANCOVA): Analysis of covariance is a method for adjusting the groups for the initial readings (baseline) of selected variables in our study and then seeing the effect of the treatments (materials) such as CollaPlug and PRF on these interested variables. If we had not measured the interested variables in our study before the start of treatment, i.e., baseline readings, we would have come to entirely the wrong conclusion about the impact of treatment on post-ARP test readings. The ANCOVA was carried out taking $5 \%$ as level of significance.

\section{CLINICAL PARAMETER}

\section{Buccal Crestal Bone Level}

- Descriptive statistics of buccal crestal bone level (Table 1 and Graph 1).

- The baseline, post-ARP, and adjusted (for baseline) means of buccal crestal bone level among the CollaPlug group and PRF group. The baseline mean is $4.67 \pm 0.54$ for CollaPlug group and $5.43 \pm 0.47$ for PRF group. The postmean is $6.98 \pm 0.60$ for CollaPlug group and $6.93 \pm$ 0.55 for PRF group. The adjusted (for baseline) mean is $7.30 \pm 0.411$ for CollaPlug group and $6.61 \pm 0.411$ for PRF group. From the graph, we can make out that there is increase in the adjusted (for baseline) mean when compared with post-PRP mean in CollaPlug, whereas it has decreased in PRF (Table 2 and Graph 2).

- Analysis of covariance for buccal crestal bone level among two groups indicates that there is significant main effect ( $p$-value $<0.05$ ) for treatment, i.e., post-ARP readings differ significantly across the two groups. However, a significant relationship exists between post and baseline readings. So, it can be said that when we statistically control for baseline readings,

Table 2: Means of buccal crestal bone level among the four groups in baseline, post, and adjusted posttests

\begin{tabular}{lllll}
\hline Treatment & $N$ & $\begin{array}{l}\text { Baseline } \\
\text { mean }\end{array}$ & Post-mean & $\begin{array}{l}\text { Adjusted } \\
\text { mean }\end{array}$ \\
\hline CollaPlug & 10 & 4.67 & 6.98 & 7.30 \\
PRF & 10 & 5.43 & 6.93 & 6.61 \\
\hline
\end{tabular}

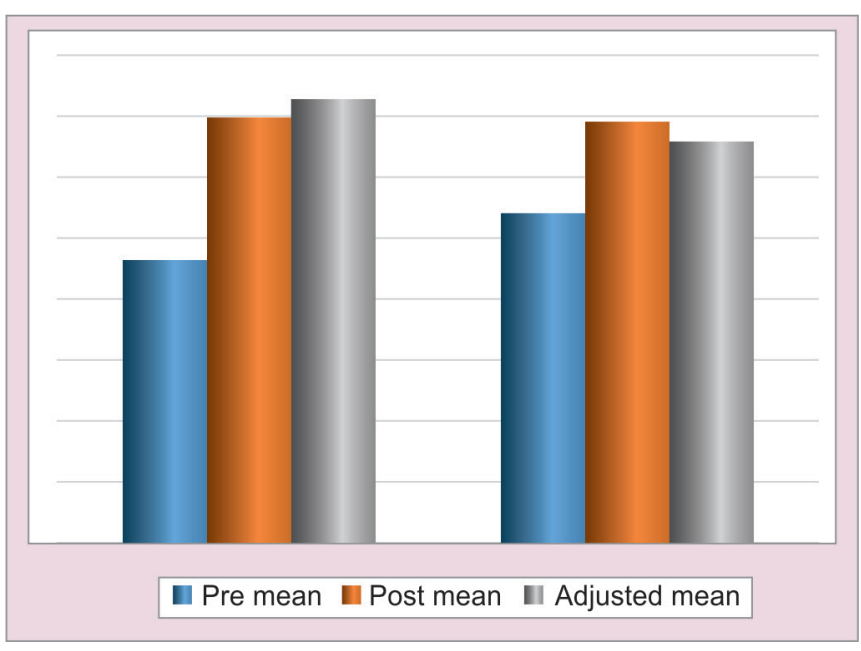

Graph 2: Multiple barplot of means of buccal crestal bone level among the two groups in baseline, post, and adjusted posttests 
Table 3: Analysis of covariance for buccal crestal bone level among two groups

\begin{tabular}{llllll}
\hline Source & $\begin{array}{l}\text { Sum of } \\
\text { squares }\end{array}$ & $d f$ & $\begin{array}{l}\text { Mean } \\
\text { square }\end{array}$ & $F$ & $p$-value \\
\hline $\begin{array}{l}\text { Baseline vertical } \\
\text { buccal }\end{array}$ & 32.06 & 1.00 & 32.06 & 19.63 & 0.00 \\
Group & 18.22 & 2.00 & 9.11 & 5.58 & 0.01 \\
Error & 27.77 & 17.00 & 1.63 & & \\
\hline
\end{tabular}

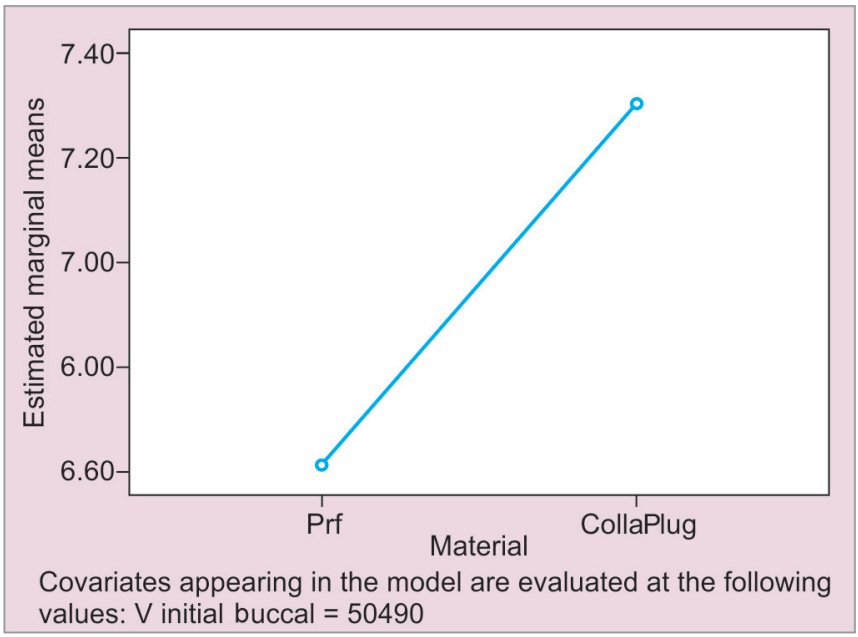

Graph 3: Profile plot for estimated marginal means of post-ARP for buccal crestal bone level

treatment has influence on post-ARP readings. That is, CollaPlug group and PRF group have significantly different mean post-ARP. Estimated marginal means of post-ARP show that there is less resorption in the buccal crestal bone in the PRF group when compared with the CollaPlug group (Table 3 and Graph 3).

\section{DISCUSSION}

The resorption of the alveolar ridge following tooth extraction is a physiological phenomenon. The preservation of the morphology of the extraction socket has become critical due to increase in demand for implantsupported prosthetic restoration. In the field of implant dentistry, the goal is to increase bone volume in order to accommodate implant and thus improve implant stability. Bone graft materials have played an important role in regenerative dentistry for many years.

There are three distinct properties of graft materials: Osteogenicity (ability to directly deposit bone by the viable osteoblasts present in the bone graft), osteoconductivity (ability of the graft to act as passive scaffolding that supports new bone formation and ingrowth of capillaries), and osteoinductivity (ability of differentiating factors that facilitate the recruitment and differentiation of mesenchymal stem cells and specifically induce them to form osteoblasts which deposit the new bone).
Among these the focus is on the osteoinductive property. The osteoinductive bone grafts contain the growth factors responsible for these stimuli, which are of the family of bone morphogenetic proteins, transforming growth factor (TGF- $\alpha$ ), insulin-like growth factor (IGF), fibroblast growth factor (FGF), platelet-derived growth factor (PDGF), and epidermal growth factor.

Today's concept in tooth extraction shall routinely consider maintenance of the existing extraction socket dimensions with some sort of bone-replacement material. This procedure has been called ARP or preservation.

Traditionally, ARP includes the use of particulate alloplasts, xenografts, autografts, allografts, and membranes manufactured from various materials, including those that are bioabsorbable or nonresorbable, naturally derived or synthetic. Most of these materials have been shown not only to be osteoconductive but also many of them are associated with a number of disadvantages, such as increased overall cost, the requirement for a second surgical site, and the use of animal-derived products. ${ }^{9-11,19,20}$

Till date, the rich and readily available autologous source of growth factors is from platelets derived from the peripheral blood. They contain a number of different growth factors which are released into the tissue after injury. These include TGF- $\alpha$, PDGF, IGF, and FGF, which act as differential factors on regenerating tissues. The PDGF is angiogenic and is known to stimulate the reproduction and chemotaxis of connective tissue cells and matrix deposition. ${ }^{21}$

Though PRP was the first platelet concentrate to be invented, its preparation involved biochemical handling and the use of potential immunogenic material; hence, PRP is not a strict autologous material. ${ }^{22,23}$

Choukroun's PRF is a simple strategy of concentrating platelets or enriching blood clot which acts as a bioscaffold and reservoir of growth factors for tissue regeneration. It is strictly autologous and involves no biochemical handling. ${ }^{24}$

A different approach for socket preservation is the use of bioabsorbable collagen. Collagen is the most abundant extracellular matrix protein and component of connective tissue. The collagen used in dental procedures is readily isolated and purified from various animal species by enzyme treatment. Collagen type I is the main organic component that is originally secreted by osteoblasts, which then becomes mineralized at a later stage of bone development. Collagen has been actively investigated as a favorable artificial environment for bone in-growth. It was shown that endothelial cells adhere, spread, and proliferate on a collagen membrane. CollaPlug, an absorbable collagen sponge, consists of 85 to $95 \%$ bovine collagen type I and 5 to $15 \%$ bovine collagen type III and has been 
used with great success. The sponge serves as a support to prevent the collapse of the surrounding soft tissue into the socket during the healing process. ${ }^{14,25}$ Hence, the present clinical study was done to compare the clinical effectiveness of Choukroun's PRF and CollaPlug (Zimmer) in preserving the dimensions of extraction socket during the healing period of 4 months.

The subjects were in the age group of $24.7 \pm 1.3$ years. The gender distribution was similar between groups.

The patients in this study were nonsmokers. Tobacco usage was found to negatively affect bone grafting procedures. Nonsmokers were selected in order to avoid a factor that could compromise or interfere with the normal healing of bone graft. ${ }^{26-28}$

Four months time frame seems to be standard for evaluating the success of socket preservation/ARP. In this study, the healing time after ARP and before implant placement was 4 months. This schedule was assigned following a standard protocol where a 4- to 6-month period is recommended., ${ }^{9}$-31

Extraction sockets are considered a reliable model for evaluation of bone healing. ${ }^{32}$ To promote a fair comparison between groups, special attention was given in this study to site selection during subject recruitment. Only maxillary incisors were included to evaluate extraction sockets of similar size and anatomy, thus minimizing variables and strengthening the result obtained.

Another factor that may have an effect on the bone remodeling process after ARP is flap elevation. Flap elevation often results in poor blood supply, more bone loss, delayed wound healing and compromises soft tissue appearance. Previous study showed that the order of magnitude of bone resorption caused by flap reflection alone is only about $1 \mathrm{~mm} .{ }^{33}$ Fickl et $\mathrm{al}^{34}$ demonstrated that during a 3-month period of healing after elevation of mucosal flaps for tooth extraction, soft and hard tissue reduction was $14 \%$ more than "flapless" exodontia. Hence, flapless exodontias were our protocol.

The buccal crestal bone level in the CollaPlug group had a baseline mean of $4.67 \pm 0.54$ and post-mean of $6.98 \pm 0.60$, whereas in the PRF group, baseline mean was $5.43 \pm 0.47$ and post-mean was $6.93 \pm 0.55$. The bone resorption was found in both the groups $(2.31 \mathrm{~mm}$ for CollaPlug and $1.5 \mathrm{~mm}$ for PRF) in agreement with previous studies. ${ }^{9,35-37}$ However, there was increased bone loss in CollaPlug group compared with the PRF group, which was found statistically significant.

The practical aspect of Choukroun's PRF use in clinical practice needs to be examined carefully. As the autologous PRF preparation utilizes the patient's own blood, the risk of human to human disease is virtually eliminated, making it a safe treatment modality. Preparation of PRF, however, adds another step to the surgical procedure and increases the time for procedure by 10 to 20 minutes. PRF preparation can be performed by a properly trained surgical assistant under the supervision of a surgeon.

In the present study, PRF and CollaPlug were used to preserve the socket dimension. A favorable response was observed in all the clinical parameters recorded in both the groups. In the PRF group, it exhibited the following properties and advantages: (1) it is safe because of an autologous preparation; (2) it provides adhesiveness and tensile strength for clot stabilization; (3) it is biologically acceptable; (4) it contains important growth factors, such as PDGF and TGF- released by platelets; (5) it promotes angiogenesis; (6) it contains a dense fibrin network that is highly osteoconductive; (7) it has hemostatic properties; (8) it contains high concentrations of leukocytes, which reduces the risk of infections; (9) it improves wound healing and is also an affordable treatment modality.

For all of the reasons mentioned above and from the results we obtained in our study treated with PRF, our clinical impression is that it significantly enhances socket healing.

\section{CONCLUSION}

The following conclusions were drawn from the present study:

There was significantly larger buccal crestal bone loss in CollaPlug group compared with the PRF group, which was found statistically significant.

Within the limits of the present study, the two tested socket preservation materials seem to be effective in the treatment of extraction sockets, even though the design of the study did not allow us to evaluate to what extent the clinical improvement could be attributed to the PRF per se, since a negative control was not included in this investigation. However, preparation of PRF is not very cumbersome and inexpensive, which makes it a better socket preservation material than CollaPlug. Also, further studies should be carried out by taking control sites to confirm the effects of PRF in bone regeneration and need to be corroborated by investigations on larger population.

\section{REFERENCES}

1. Hoffmann O, Bartee BK, Beaumont C, Kasaj A, Deli G, Zafiropoulos GG. Alveolar bone preservation in extraction sockets using non-resorbable dPTFE membranes: a retrospective non-randomized study. J Periodontol 2008 Aug;79(8):1355-1369.

2. Boyne PJ. Osseous repair of the postextraction alveolus in man. Oral Surg Oral Med Oral Pathol 1966 Jun;21(6):805-813.

3. Amler $\mathrm{MH}$. The time sequence of tissue regeneration in human extraction wounds. Oral Surg Oral Med Oral Pathol 1969 Mar;27(3):309-318.

4. Lin WL, McCulloch CA, Cho MI. Differentiation of periodontal ligament fibroblasts into osteoblasts during socket 
healing after tooth extraction in the rat. Anat Rec 1994 Dec;240(4):492-506.

5. Araujo MG, Sukekava F, Wennstrom JL, Lindhe J. Ridge alterations following implant placement in fresh extraction sockets: an experimental study in the dog. J Clin Periodontol 2005 Jun;32(6):645-652.

6. Schropp L, Wenzel A, Kostopoulos L, Karring T. Bone healing and soft tissue contour changes following single-tooth extraction: a clinical and radiographic 12 -month prospective study. Int J Periodontics Restorative Dent 2003 Aug;23(4): 313-323.

7. Evian CI, Rosenberg ES, Coslet JG, Corn H. The osteogenic activity of bone removed from healing extraction sockets in humans. J Periodontol 1982 Feb;53(2):81-85.

8. Quinn JH, Kent JN. Alveolar ridge maintenance with solid nonporous hydroxylapatite root implants. Oral Surg Oral Med Oral Pathol 1984 Nov;58(5):511-521.

9. Iasella JM, Greenwell H, Miller RL, Hill M, Drisko C, Bohra AA, Scheetz JP. Ridge preservation with freeze-dried bone allograft and a collagen membrane compared to extraction alone for implant site development: a clinical and histologic study in humans. J Periodontol 2003 Jul;74(7):990-999.

10. Bartee BK. Extraction site reconstruction for alveolar ridge preservation. Part 2: membrane-assisted surgical technique. J Oral Implantol 2001;27(4):194-197.

11. Darby I, Chen S, De Poi R. Ridge preservation: what is it and when should it be considered. Aust Dent J 2008 Mar;53(1): 11-21.

12. Serino G, Biancu S, Iezzi G, Piattelli A. Ridge preservation following tooth extraction using a polylactide and polyglycolide sponge as space filler: a clinical and histological study in humans. Clin Oral Implants Res 2003 Oct;14(5):651-658.

13. Sunitha Raja V, Muniratnam Naidu E. Platelet-rich fibrin: evolution of a second-generation platelet concentrate. Indian J Dent Res 2008 Jan-Mar;19(1):42-46.

14. Marx RE, Carlson ER, Eichstaedt RM, Schimmele SR, Strauss JE, Georgeff KR. Platelet-rich plasma growth factor enhancement for bone grafts. Oral Surg Oral Med Oral Pathol Oral Radiol Endod 1998 Jun;85(6):638-646.

15. Dohan DM, Choukroun J, Diss A, Dohan SL, Dohan AJ, Mouhyi J, Gogly B. Platelet-rich fibrin (PRF): a secondgeneration platelet concentrate. Part I: technological concepts and evolution. Oral Surg Oral Med Oral Pathol Oral Radiol Endod 2006 Mar;101(3):e37-e44.

16. Dohan DM, Choukroun J, Diss A, Dohan SL, Dohan AJ, Mouhyi J, Gogly B. Platelet-rich fibrin (PRF): a secondgeneration platelet concentrate. Part II: platelet-related biologic features. Oral Surg Oral Med Oral Pathol Oral Radiol Endod 2006 Mar;101(3):e45-e50.

17. Dohan DM, Choukroun J, Diss A, Dohan SL, Dohan AJ, Mouhyi J, Gogly B. Platelet-rich fibrin (PRF): a secondgeneration platelet concentrate. Part III: leukocyte activation: a new feature for platelet concentrates? Oral Surg Oral Med Oral Pathol Oral Radiol Endod 2006 Mar;101(3):e51-e55.

18. Choukroun J, Diss A, Simonpieri A, Girard MO, Schoeffler C, Dohan SL, Dohan AJ, Mouhyi J, Dohan DM. Platelet-rich fibrin (PRF): a second-generation platelet concentrate. Part IV: clinical effects on tissue healing. Oral Surg Oral Med Oral Pathol Oral Radiol Endod 2006 Mar;101(3):e56-e60.

19. Nemcovsky CE, Serfaty V. Alveolar ridge preservation following extraction of maxillary anterior teeth. Report on 23 consecutive cases. J Periodontol 1996 Apr;67(4):390-395.
20. Sclar A. Ridge preservation technique for optimal esthetics and function: the Bio-Col technique. Compend Contin Educ Dent 1999;6(Suppl):3-11.

21. Bowen-Pope DF, Vogel A, Ross R. Production of plateletderived growth factor-like molecules and reduced expression of platelet-derived growth factor receptors accompany transformation by a wide spectrum of agents. Proc Natl Acad Sci U S A 1984 Apr;81(8):2396-2400.

22. Sanchez AR, Sheridan PJ, Kupp LI. Is platelet-rich plasma the perfect enhancement factor? A current review. Int J Oral Maxillofac Implants 2003 Jan-Feb;18(1):93-103.

23. Hamdan AA, Loty S, Isaac J, Bouchard P, Berdal A, Sautier JM. Platelet-poor plasma stimulates proliferation but inhibits differentiation of rat osteoblastic cells in vitro. Clin Oral Implants Res 2009 Jun;20(6):616-623.

24. Kang YH, Jeon SH, Park JY, Chung JH, Choung YH, Choung HW, Kim ES, Choung PH. Platelet-rich fibrin (PRF) is a bioscaffold and reservoir of growth factors for tissue regeneration. Tissue Eng Part A 2011 Feb;17(3-4):349-359.

25. Feuille F, Knapp CI, Brunsvold MA, Mellonig JT. Clinical and histologic evaluation of bone-replacement grafts in the treatment of localized alveolar ridge defects. Part 1: mineralized freeze-dried bone allografts. Int J Periodontics Restorative Dent 2003 Feb;23(1):29-35.

26. Haber J, Kent RL. Cigarette smoking in a periodontal practice. J Periodontol 1992 Feb;63(2):100-106.

27. Riebel GD, Boden SD, Whitesides TE, Hutton WC. The effect of nicotine on incorporation of cancellous bone graft in an animal model. Spine (Phila Pa 1976) 1995 Oct;20(20):2198-2202.

28. Levin L, Schwartz-Arad D. The effect of cigarette smoking on dental implants and related surgery. Implant Dent 2005 Dec;14(4):357-361.

29. Li J, Wang HL. Common implant-related advanced bone grafting complications: classification, etiology, and management. Implant Dent 2008 Dec;17(4):389-401.

30. Morishita M, Miyagi M, Iwamoto Y. Effects of sex hormones on production of interleukin-1 by human peripheral monocytes. J Periodontol 1999 Jul;70(7):757-760.

31. Adell R. Tissue integrated prostheses in clinical dentistry. Int Dent J 1985 Dec;35(4):259-265.

32. Becker W, Clokie C, Sennerby L, Urist MR, Becker BE. Histologic findings after implantation and evaluation of different grafting materials and titanium micro screws into extraction sockets: case reports. J Periodontol 1998 Apr;69(4):414-421.

33. Hammerle $\mathrm{CH}$, Schmid J, Olah AJ, Lang NP. A novel model system for the study of experimental guided bone formation in humans. Clin Oral Implants Res 1996 Mar;7(1):38-47.

34. Lang NP, Hammerle CH, Bragger U, Lehmann B, Nyman SR. Guided tissue regeneration in jawbone defects prior to implant placement. Clin Oral Implants Res 1994 Jun;5(2): 92-97.

35. Araujo MG, Lindhe J. Dimensional ridge alterations following tooth extraction. An experimental study in the dog. J Clin Periodontol 2005 Feb;32(2):212-218.

36. Araujo MG, Lindhe J. Ridge preservation with the use of Bio-Oss collagen: A 6-month study in the dog. Clin Oral Implants Res 2009 May;20(5):433-440.

37. Araujo MG, Lindhe J. Ridge alterations following tooth extraction with and without flap elevation: an experimental study in the dog. Clin Oral Implants Res 2009 Jun;20(6): 545-549. 